\title{
How Implicit Bias Contributes to Racial Disparities in Maternal Morbidity and Mortality in the United States
}

\author{
Bani Saluja, $\mathrm{MPH}^{1}$ and Zenobia Bryant, $\mathrm{PhD}^{2}$
}

\begin{abstract}
Over the past two decades, maternal mortality rates have declined around the world. In the United States, however, 700 women die each year as a result of pregnancy or delivery complications. This represents a 50\% increase in the U.S. maternal mortality rate over the same time period. According to the Centers for Disease Control and Prevention (CDC), the pregnancy-related mortality ratios vary significantly by race, with White women experiencing 13.0 deaths per 100,000 births, compared with 42.8 deaths per 100,000 births for Black women, from 2011 to 2015. Multiple studies suggest that implicit bias-defined as the attitudes or stereotypes that affect our understanding, actions, and decisions in an unconscious manner-is most likely a contributing factor to this alarming racial health disparity. The failure to recognize the pain of African American patients, regardless of whether it is conscious or unconscious, has the potential to affect the way obstetrician/gynecologists counsel patients about treatment options when it comes to chronic conditions, contraception, vaginal birth after cesarean delivery, and the management of fibroids. In this article, we will review implicit bias and the impact it can have on health care and health disparities.
\end{abstract}

Keywords: maternal morbidity, maternal mortality, implicit bias, racism

\section{Introduction}

O VER THE PAST two decades, maternal morbidity and mortality (MMM) rates have declined around the world. However, MMM rates have been increasing steadily in the United States - the only developed country in which this has occurred. ${ }^{1}$ Each year, $\sim 700$ women die because of pregnancy or delivery complications in the United States, but approximately three in five pregnancy-related deaths could have been prevented. $^{2}$ Furthermore, pregnancy-related mortality rates vary significantly by race. According to data from the Centers for Disease Control and Prevention (CDC) National Pregnancy Mortality Surveillance System (PMSS) spanning 2007-2016, White women experienced 12.7 deaths per 100,000 births compared with 40.8 deaths per 100,000 births for Black women. ${ }^{3}$ Understanding what contributes to this racial divide in maternal health outcomes is of vital importance because it can illuminate where and how to tackle such a multifaceted issue and focus the scope of public health prevention programs.
Decades of research indicate that this serious U.S. public health problem involves structural racism and its negative effects on the minds and bodies of all racial groups, especially people of color. ${ }^{4}$ Structural racism refers to the summative ways in which societies foster racial discrimination through mutually reinforcing systems such as housing, education, media, employment, and health care. ${ }^{5}$ Today, an imbalance of inherited resources and persisting discrimination restrict the access of many Americans of color to better jobs, quality education, political power, healthy neighborhoods, and quality health care. ${ }^{4}$ As a result, implicit, discriminatory attitudes, and behaviors have permeated the U.S. health care system and likely are a powerful contributing factor to the high negative maternal health outcomes experienced by Black women.

\section{What Is Implicit Bias?}

Implicit bias is different from overt and intentional discrimination and develops early in life from exposure to

\footnotetext{
${ }^{1}$ Department of Behavioral and Community Health, University of Maryland School of Public Health, College Park, Maryland, USA.

${ }^{2}$ Scientific Consulting Group, Inc., Gaithersburg, Maryland, USA.

(C) Bani Saluja and Zenobia Bryant 2021; Published by Mary Ann Liebert, Inc. This Open Access article is distributed under the terms of the Creative Commons Attribution Noncommercial License (http://creativecommons.org/licenses/by-nc/4.0/) which permits any noncommercial use, distribution, and reproduction in any medium, provided the original author(s) and the source are cited.
}

Correction added on February 19, 2021 after first online publication of November 25, 2020: The article reflects Open Access, with copyright transferring to the author(s), and a Creative Commons Attribution Noncommercial License (CC-BY-NC) added (http:// creativecommons.org/licenses/by-nc/4.0/). 
repeated reinforcement of stereotypes perpetuated by structural discrimination. ${ }^{6}$ The mere existence of cultural stereotypes regarding certain social or racial groups has the potential to influence one's behavior toward individuals from the stereotyped group. ${ }^{6}$ Implicit bias is defined as thoughts and feelings that exist outside of conscious awareness and subsequently can affect human understanding, actions, and decisions unknowingly. ${ }^{7,8}$ These biases cause attitudes about other people based on personal characteristics, including, but not limited to, age, race, and ethnicity. ${ }^{8}$ Because implicit bias is unconscious, it can be difficult to measure, and actions resulting from it often are challenging to recognize and control. ${ }^{7}$

\section{Measuring Implicit Bias}

Implicit bias cannot be measured well with standard (selfreport) survey questions. ${ }^{9}$ The most common tool used to assess implicit bias is the Implicit Association Test (IAT). This tool is available online and is a computerized, timed dual categorization task that measures implicit preferences by bypassing conscious processing. ${ }^{6,8}$ The IAT has been used in hundreds of studies across a variety of disciplines, including psychology, health, political science, and market research. ${ }^{9}$ Demonstrations of the IAT can be found at https://implicit.harvard.edu.

\section{Implicit Bias, Health Care Providers, and Maternal Health}

Implicit bias is directly correlated with lower quality of care for patients and may be activated under stressful working conditions. ${ }^{7,10}$ The potential influence of implicit bias is especially relevant in settings that are prone to overload or high stress. These environments include emergency departments or labor and delivery settings, where relying on automatic or unconscious processes to execute medical decision making quickly becomes essential. However, such automatic processes also are likely to activate stereotypes and unconscious beliefs. ${ }^{8}$ In addition, cognitive stressors, such as overcrowding and the demand to care for more patients during a shift, are associated with an increase in implicit bias. ${ }^{11}$

Although the medical profession strives for equal treatment of all patients, racial disparities in health care are prevalent. ${ }^{6}$ Racial disparities exist because implicit bias affects health care providers' perceptions and decisions, creating inequalities in access, patient-provider interactions, treatment decisions, and health outcomes. ${ }^{4,12}$ Even when health care providers do not display explicit discrimination, implicit biases still exist. ${ }^{4} \mathrm{~A}$ 2012 study found that, after adjusting for medically necessary procedures, cesarean deliveries were more common among Black and Latina women than White women. Cesarean deliveries lead to more negative health outcomes for both mother and baby; research on obstetric care increasingly considers rising $\mathrm{C}$-section rates to be a source of MMM. Three of the six leading causes of maternal mortality are associated with cesareans: hemorrhage, complications of anesthesia, and infection. ${ }^{13}$

Moreover, many health care providers struggle to acknowledge the impact of personal implicit bias on how they care for their patients. ${ }^{14}$ A study conducted by the Society for Maternal Fetal Medicine showed inconsistency between clinicians' willingness to acknowledge disparities in their practice and their consideration of implicit bias. In fact, $84 \%$ of respondents agreed that disparities affect their practice, but only $29 \%$ believed that personal biases influenced their ability to care for patients. ${ }^{8}$

Furthermore, some health care providers still hold false beliefs about biological differences between Black and White individuals that increase implicit bias. These beliefs include ideas that Black people have less sensitive nerve endings, thicker skin, and stronger bones. ${ }^{15}$ The presence of these beliefs causes health care providers to rate Black patients' pain lower and results in less-appropriate treatment recommendations. ${ }^{15}$ Specifically regarding pain management, Rust et al. found significant racial/ethnic differences in the rates of epidural analgesia. Minorities, specifically Black non-Hispanic and Hispanic patients, had significantly lower rates of epidural analgesia. ${ }^{16}$ As a result, health care provider bias, although unintentional and often unrecognized, does lead to mistrust in the health care system and poor patient satisfaction from people of color. ${ }^{17}$

In a comprehensive report entitled Birth Settings in America: Outcomes, Quality, Access, and Choice by the National Academies of Sciences, the authors reference the Listening to Mothers III survey, which found that approximately one in five Black and Hispanic women experienced mistreatment from hospital-based care providers because of their race, ethnicity, cultural background, and/or language. ${ }^{18}$ As reported in an article published in 2018, anthropologist Dana-Ain Davis analyzed the birth stories of Black women living in the United States. These birth stories describe various forms of maltreatment during medical encounters while they were pregnant or during labor and delivery. Consequently, some women avoided the hospital if possible and utilized midwives and doulas for home or birth center services. ${ }^{19}$

\section{The Effects of Implicit Bias on Patient-Provider Communication}

In addition to its potential impact on medical decision making, implicit bias also can affect how providers communicate with patients. Studies found that people of color are more likely to report lower satisfaction with health care provider interactions. ${ }^{7}$ Subtle racial biases may be expressed in such ways as approaching patients with a condescending tone that decreases the likelihood that patients will feel heard and valued by their providers or recommending different treatment options for patients based on assumptions about their treatment adherence capabilities or presumed health conditions. ${ }^{7}$ Consistent and robust evidence shows that health care providers who exhibit higher implicit bias demonstrate higher verbal dominance in their communication styles and less interpersonal treatment. ${ }^{11}$ Subsequently, patients of these providers report poorer satisfaction ratings and greater difficulty understanding or following recommendations, which can perpetuate biases held by the provider. ${ }^{11}$ This is a vicious cycle that highlights the importance of improved communication and the need for health care providers to be aware of their own implicit biases.

The actions of providers and their interactions with patients are highly associated with racial disparities in women's experience of trauma during birth. In the United States, 30\% of Black and Hispanic women who delivered in hospitals reported provider mistreatment, whereas only $21 \%$ of White women reported provider mistreatment. ${ }^{12}$ The perceived dismissals women felt of legitimate concerns and symptoms, such as 
preeclampsia and hypertension, can help explain the existence of poor birth outcomes even for Black women with the most advantages, such as high income and advanced education. ${ }^{20}$

\section{Opportunities for Intervention}

Efforts to resolve racial disparities in maternal health are constrained by two main challenges: (1) lack of reliable data on patient identity (including race, ethnicity, native country, and language) and (2) limited patient and staff education on the best practices for collecting information related to identity. ${ }^{8}$ The improvement of cultural humility programming for health care providers is increasingly emphasized. Cultural humility goes beyond cultural competency and is defined as a life-long commitment to self-evaluation and self-critique in an effort to address power imbalances and advocate for others. ${ }^{21}$ Practicing cultural humility mitigates implicit bias, promotes empathy, and aids health care providers in acknowledging and respecting patients' individuality. ${ }^{21} \mathrm{Cul}-$ tural humility principles emphasize that providers should aim to connect with patients instead of assuming expertise on the patient's race, culture, or ethnicity and how those relate to the patient's health. ${ }^{21}$ Efforts to incorporate awareness of implicit bias in medical and nursing school and residency training programs are important steps in training the next generation of health care providers to treat patients and families with respect and provide high-quality care to an increasingly diverse patient population.

Evidence also shows that mindfulness interventions are effective at reducing implicit bias. Mindfulness-based practices can offer additional benefits to health care providers, including decreasing burnout and improving empathy and well-being. ${ }^{11}$ In one successful intervention, after completing a baseline IAT and an educational program about the impact of implicit bias on discriminatory behaviors, participants were trained on how to apply several bias-reduction strategies to everyday practice. These strategies included (1) stereotype replacement, in which individuals were trained to recognize stereotypes being perpetuated in society and within themselves and how to replace them with nonstereotypic responses; (2) counter-stereotypic imaging, in which individuals imagine someone from a marginalized group who does not fulfill commonly believed stereotypes about that group; (3) individuation, in which individuals try to get to know someone else and focus on their individual characteristics, instead of their group-based characteristics; and (4) perspective taking, in which individuals consciously assess a situation from the viewpoint of another racial group. Furthermore, health care providers should actively seek out as many opportunities for cross-cultural contact as possible throughout their career. ${ }^{11}$

As for opportunities regarding patient empowerment, patients who read their clinical notes through online patient portals report that doing so engages them actively in their care, improves their sense of control over their health, and enhances safety, especially among minority patients. ${ }^{22}$ Qualitative research also suggests that reading their clinical notes can help patients feel listened to, validated, and understood. ${ }^{22}$ Open notes may increase trust between patients and clinicians, reduce transmission of biases, and increase patient engagement. Also, patients should be given information to make informed decisions about continuing or ending a relationship with a provider; expecting mothers are not obligated to remain with a health care provider throughout a pregnancy and should be encouraged to find the right fit, particularly early in the pregnancy. ${ }^{19}$

Additionally, hospital systems can implement mandatory MMM reviews, which help to identify areas of substandard care that need improvement. ${ }^{14}$ If conducting a systematic, multidisciplinary review of all cases of maternal death and severe morbidity and establishing a mechanism to disseminate knowledge gained from those reviews became standard practice, then hundreds of lives could be saved.

Furthermore, the disparities of any condition, let alone maternal health, cannot be discussed without also discussing the system-level factors that may contribute to them. An important system-level factor that impacts clinical care is the availability of transportation to in-person visits. Cab vouchers or other transit programs may help with this barrier. The frequency of scheduled in-person visits poses another challenge; innovations in telehealth may assist in improved patient-centered care. Also, for women who do not speak English, failures in adequate communication also can contribute to maternal health disparities. Increasing the availability of interpreter services, both in person and on the phone, could support improvements in this area. ${ }^{14}$

\section{Conclusion}

To reiterate, most pregnancy-related deaths can be prevented; all levels of the social/ecological model should be considered together when examining racial disparities in health care. Further identification and evaluation of factors contributing to racial disparities is crucial to informing and implementing prevention strategies that will effectively reduce disparities in pregnancy-related mortality, including strategies to improve women's health and access to quality care in all maternal phases: preconception, pregnancy, and postpartum. ${ }^{3}$ We recognize that addressing this complex national problem requires coordination and collaboration among patients, families, health care providers, community organizations, hospitals, health systems, and policymakers. ${ }^{3}$ We believe the interdisciplinary work can be done, but individual implicit bias and the deep impact of structural racism must be acknowledged and accepted before real progress can be made in reducing racial disparities in maternal mortality and decreasing maternal deaths overall in the United States.

\section{Author Disclosure Statement}

No competing financial interests exist.

\section{Funding Information}

No funding was received for this article.

\section{References}

1. Delbanco S, Lehan M, Montalvo T, Levin-Scherz J. The rising U.S. maternal mortality rate demands action from employers. Harvard Business Review. Published online June 28, 2019. Available at: https://hbr.org/2019/06/therising-u-s-maternal-mortality-rate-demands-action-fromemployers Accessed May 20, 2020.

2. Petersen EE, Davis NL, Goodman D, et al. Vital signs: Pregnancy-related deaths, United States, 2011-2015, and strategies for prevention, 13 states, 2013-2017. MMWR Morb Mortal Wkly Rep 2019;68:423-429. 
3. Petersen EE. Racial/ethnic disparities in pregnancy-related deaths-United States, 2007-2016. MMWR Morb Mortal Wkly Rep 2019;68:762-765.

4. Feagin J, Bennefield Z. Systemic racism and U.S. health care. Soc Sci Med 2014;103:7-14.

5. Bailey ZD, Krieger N, Agénor M, Graves J, Linos N, Bassett MT. Structural racism and health inequities in the USA: Evidence and interventions. Lancet 2017;389:1453-1463.

6. Chapman EN, Kaatz A, Carnes M. Physicians and implicit bias: How doctors may unwittingly perpetuate health care disparities. J Gen Intern Med 2013;28:1504-1510.

7. Hall WJ, Chapman MV, Lee KM, et al. Implicit racial/ ethnic bias among health care professionals and its influence on health care outcomes: A systematic review. Am J Public Health 2015;105:e60-e76.

8. Howell EA, Brown H, Brumley $\mathrm{J}$, et al. Reduction of peripartum racial and ethnic disparities: A conceptual framework and maternal safety consensus bundle. Obstet Gynecol 2018;131:770-782.

9. Blair IV, Steiner JF, Havranek EP. Unconscious (implicit) bias and health disparities: Where do we go from here? Perm J 2011;15:71-78.

10. Hilton EJ, Goff KL, Sreedharan R, Lunardi N, Batakji M, Rosenberger DS. The flaw of medicine: Addressing racial and gender disparities in critical care. Anesthesiol Clin 2020;38:357-368.

11. Johnson TJ. Racial bias and its impact on children and adolescents. Pediatr Clin North Am 2020;67:425-436.

12. Vedam S, Stoll K, Taiwo TK, et al. The Giving Voice to Mothers study: Inequity and mistreatment during pregnancy and childbirth in the United States. Reprod Health 2019;16:77.

13. Roth LM, Henley MM. Unequal motherhood: Racial-ethnic and socioeconomic disparities in cesarean sections in the United States. Soc Probl 2012;59:207-227.

14. Jain JA, Temming LA, D'Alton ME, et al. SMFM special report: Putting the "M" back in MFM: Reducing racial and ethnic disparities in maternal morbidity and mortality: A call to action. Am J Obstet Gynecol 2018;218:B9-B17.

15. Jones SCT, Anderson RE, Gaskin-Wasson AL, Sawyer BA, Applewhite K, Metzger IW. From "crib to coffin": Navigating coping from racism-related stress throughout the lifespan of black Americans. Am J Orthopsychiatry 2020; 90:267-282.
16. Rust G, Nembhard WN, Nichols M, et al. Racial and ethnic disparities in the provision of epidural analgesia to Georgia Medicaid beneficiaries during labor and delivery. Am J Obstet Gynecol 2004;191:456-462.

17. Alhusen JL, Bower KM, Epstein E, Sharps P. Racial discrimination and adverse birth outcomes: An integrative review. J Midwifery Womens Health 2016;61:707-720.

18. National Academies of Science, Engineering, and Medicine. Birth settings in America: Outcomes, quality, access, and choice. National Academies Press, 2020.

19. Davis D-A. Obstetric racism: The racial politics of pregnancy, labor, and birthing. Med Anthropol 2019;38:560-573.

20. Villarose L. Why America's black mothers and babies are in a life-or-death crisis. The New York Times. Published April 11, 2018. www.nytimes.com/2018/04/11/magazine/ black-mothers-babies-death-maternal-mortality.html Accessed May 26, 2020.

21. Masters C, Robinson D, Faulkner S, Patterson E, McIlraith $\mathrm{T}$, Ansari A. Addressing biases in patient care with the $5 \mathrm{Rs}$ of cultural humility: A clinician coaching tool. J Gen Intern Med 2019;34:627-630.

22. Blease C, Fernandez L, Bell SK, Delbanco T, DesRoches C. Empowering patients and reducing inequities: Is there potential in sharing clinical notes? BMJ Qual Saf 2020;29:1-2.

Address correspondence to: Bani Saluja, $\mathrm{MPH}$

Department of Behavioral and Community Health University of Maryland School of Public Health College Park, MD 20742

USA

E-mail: bsaluja@umd.edu

Zenobia Bryant, PhD Scientific Consulting Group, Inc. 656 Quince Orchard Road

Suite 210

Gaithersburg, MD 20878

USA

E-mail: zenobia.j.bryant@gmail.com 